\title{
Direct Surface-enhanced Raman Scattering Analysis of DNA Duplexes
}

\author{
Luca Guerrini, ${ }^{[a, b] *}$ Željka Krpetić, ${ }^{[c, d]}$ Danny van Lierop, ${ }^{[d]}$ Ramon A. Alvarez-Puebla, ${ }^{[a, b, e]}$ and Duncan \\ Graham. ${ }^{[d]}$
}

Abstract: The exploring of the genetic information carried by DNA has become a major scientific challenge. Routine DNA analysis, such as PCR, still suffers from intrinsic important limitations. Surface-enhanced Raman spectroscopy (SERS) has emerged as an outstanding opportunity for the development of DNA analysis but the application to duplexes (dsDNA) has been largely hampered due to reproducibility and/or sensitivity issues. Here we show a simple strategy to perform ultrasensitive direct label-free analysis of unmodified IsDNA with the means of SERS by using positivelycharged silver colloids. Electrostatic adhesion of DNA promotes nanoparticle aggregation into stable clusters yielding intense and reproducible SERS spectra at nanogram level. As potential applications, we report the quantitative recognition of hybridization events as well as the first examples of SERS recognition of single base mismatches and base methylations (5-methylated cytosine and N6-methylated Adenine) in duplexes.

DNA is the custodian of the memory of life. Although very stable, it is subject to modifications which are associated with evolution but more frequently with genetic diseases or cancer ${ }^{[1]}$ Due to the small content of DNA in cells and its structural complexity, its analysis require denaturation (heat), fragmentation (restriction endonucleases), separation (electrophoresis), amplification (polymerase chain reaction, PCR) and detection (quantitative PCR or microarray techniques). ${ }^{[2]}$ Besides the cost both in time and money, during these processes samples are subject to alterations ${ }^{[3]}$ and losses of epigenetic information. ${ }^{[4]}$ The advent of nanophotonics offers a unique opportunity for the development of direct, fast and ultrasensitive methods for DNA analysis. ${ }^{[5]}$ In the broad field of plasmonics, surface-enhanced Raman scattering (SERS)

[a] Dr. L. Guerrini; Prof. R.A. Alvarez-Puebla Departamento de Química Fisica e Inorganica Universitat Rovira i Virgili and Centro de Tecnologia Química Carrer de Marcel-lí Domingo s/n, 43007 Tarragona, Spain. E-mail: luca.guerrini@ctqc.org

[b] Dr. L. Guerrini; Prof. R.A. Alvarez-Puebla Medcom Advance SA

Viladecans Bussines Park, Edificio Brasil, C/ Bertran i Musitu, 83-85, 08840 Viladecans (Barcelona), Spain.

[c] Dr. Ž. Krpetić

Department Centre for BioNano Interactions University College Dublin, Belfield, Dublin 4, Ireland

[d] Dr. Ž. Krpetić, Dr. D. van Lierop, Prof. D. Graham. Centre for Molecular Nanometrology, Pure and Applied Chemistry University of Strathclyde, 295 Cathedral St., Glasgow, G1 1XL, UK.

[e] Prof. R.A. Alvarez-Puebla

ICREA, Passeig Lluís Companys 23, 08010 Barcelona, Spain

Supporting information for this article is given via a link at the end of the document. This includes experimental section; AgNP@Sp characterization (section S1); DNA-driven aggregation of AgNP@Sp and spectral reproducibility (section S2); vibrational assignment of dsDNA SERS spectra (section S3); SERS of ss and ds sequences on silver hydroxylamine colloids (section S4); ds1 SERS spectrum and the pondered sum of the individual spectra of ss 1 and ssc (section S5); and SERS spectra of ds2, ds3 and ds4 (section S6). spectroscopy has arisen as a powerful analytical tool for detection and structural characterization of biomolecules. ${ }^{[6]}$ The large majority of SERS-based DNA detection strategies rely on the selective recognition between two complementary strands to identify a specific sequence, and the use of extrinsic Raman labels for SERS readout. ${ }^{[7]}$ However, labeling of the DNA strands requires complex and costly chemistry, and does not provide any chemical-specific information. ${ }^{[8]} \mathrm{A}$ second approach is based on the direct detection of the distinctive SERS signal from DNA strands directly adsorbed onto the nanostructured surface. This strategy shows outstanding potential in terms of sensitivity, selectivity and chemical-specific information. ${ }^{[8-9]}$ To date, however, direct SERS analysis of unmodified DNA is mainly restricted to single-stranded DNA sequences (ssDNA) since the direct contact of double-stranded sequences (dsDNA) with nanostructured metallic surfaces (negative at the physiological $\mathrm{pH}$ ) is largely hindered by the negative charge of the phosphate backbone, unless relatively high DNA concentrations are employed. ${ }^{[9 b, 9 c]}$ As a result, dsDNA SERS spectra traditionally suffer from inherent poor spectral reproducibility ${ }^{[8,9 b]}$ and/or limited sensitivity hampering the extended application of label-free SERS strategies for the study of unmodified duplexes. Thus, successful translation of the analytical potential of SERS to the direct study of dsDNA would represent a great leap forward for the full exploration of the genetic information.

To overcome this major challenge, in this study we use positively-charged silver nanoparticles coated with spermine molecules (AgNP@Sp). Spermine bound to AgNPs acts as stabilizer and, upon addition of negatively-charged DNA, promotes NP aggregation into stable particle clusters. ${ }^{[10]}$ This has important consequences for the direct SERS analysis of dsDNA. Firstly, the DNA adsorption occurs via non-specific electrostatic interaction of the phosphate groups rather than by base-specific nucleoside-metal binding. Secondly, DNA promotes the nanoparticle aggregation removing the need of external aggregating agents. On the one hand, this significantly simplifies the sensing scheme, reducing the number of variables to be controlled (type and concentration of the aggregating agent, time and order of addition, etc.). On the other hand, DNA sequences are selectively sandwiched between interparticle junctions (i.e. hot-spots ${ }^{[11]}$ ) thus maximizing the intensification of the Raman signal (Fig. 1a). Thirdly, the nanoparticle aggregation occurs fast and independently of the base composition, generating long-term stable clusters in suspension, as demonstrated by the extinction spectra illustrated in Fig. 1b. This also allows us to perform the SERS measurements after several hours upon the analyte addition (i.e. when the process of DNA adhesion has reached is equilibrium). Finally, SERS spectra are acquired in colloidal suspensions with a long working distance objective. In this experimental set-up, the recorded SERS spectra are the statistically-averaged result of a large ensemble of nanoparticle/DNA clusters in continuous Brownian motions within the scattering volume. Such averaged bulk SERS regime yields goodquality spectra with well-defined average band centers, bandwidths and relative intensities. ${ }^{[12]}$ As a result, dsDNA electrostatic adhesion onto the AgNP@Sp nanoparticles allows for the ultrasensitive 

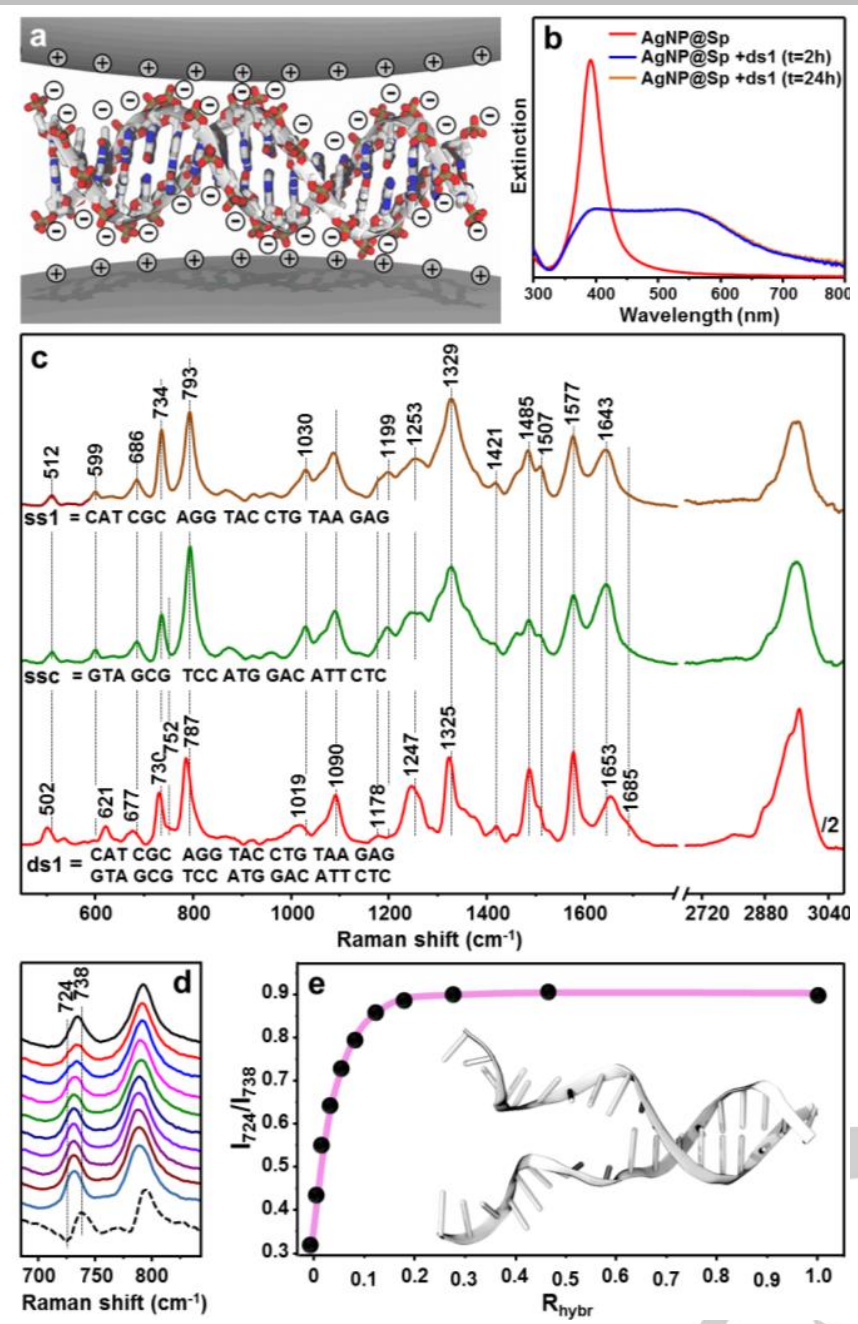

Figure 1. (a) Schematic diagram of dsDNA sandwiched between two positively charged AgNP@Sp. (b) Extinction spectra of pure AgNP@Sp colloids and in the presence of ds1 (final conc. $630 \mathrm{ng} / \mathrm{mL}$ ) acquired $2 \mathrm{~h}$ and 24h upon DNA addition (the AgNP@Sp+ds1 mixture was sonicated for few seconds before the spectral acquisition). (c) SERS spectra of ss1 and ssc (3.15 $\mu \mathrm{g} / \mathrm{mL})$, and ds1 (630 ng/mL) on AgNP@Sp. SERS spectra were normalized to the $\mathrm{PO}_{2}^{-}$stretching at $1090 \mathrm{~cm}^{-1}$. The ds 1 SERS spectrum in the $2700-3050 \mathrm{~cm}^{-1}$ region was divided by 2. (d) Detail of the $680-890 \mathrm{~cm}^{-1}$ spectral region for the SERS spectra of ssc+ds 1 mixtures at different molar ratio $R_{\text {hybr }}=[d s 1] /([d s 1]+[s s c]$ ) (from the top to the bottom: $0.011,0.024,0.041$, $0.063,0.091,0.130,0.189,0.286,0.474$ and 1$)$. The ds 1 concentration was progressively increased from 0 to $630 \mathrm{ng} / \mathrm{mL}$ while the ssc concentration was simultaneously decreased from $3.15 \mu \mathrm{g} / \mathrm{mL}$ ng to zero. The dotted line is the difference spectrum ssc-ds1 obtained by digitally subtracting the SERS spectra shown in (c). (e) Ratiometric peak intensities $\mathrm{I}_{724} / \mathrm{I}_{738}$ vs. $\mathrm{R}_{\text {hybr }}=$ $[\mathrm{ds} 1] /([\mathrm{ds} 1]+[\mathrm{ssc}])$ molar ratio.

analysis of duplexes and their modifications at the ng regime with high batch-to-batch reproducibility, yielding SERS spectra that are truly spectral representations of the nucleoside composition and organization within the helix. To confirm this, we show an ample set of examples of direct label-free SERS detection of unmodified duplexes, including quantification of hybridization events as well as recognition of single-base mismatches and base methylations (5methylated cytosine and N6-methylated adenine) which were restricted so far to single stranded sequences. ${ }^{[9 a, 9 d]}$

The average SERS spectra of two complementary singlestranded sequences (ss1 and ssc) and their corresponding doublehelix structure (ds1) on AgNP@Sp colloids are shown in Figure 1c.
The overall dsDNA concentration was kept constant throughout the entire study at ca. $630 \mathrm{ng} / \mathrm{mL}$ (corresponding to less than $3.0 \mathrm{ng}$ in the probed volume, see Section S2) and appropriately selected to yield long-term stable clusters in suspension with reproducible and unvaried SERS spectral profiles (Section S2). Vibrational assignment of dsDNA was based on literature references and comparative spectral analysis with homo- and bi-polymeric sequences (Section S3). In contrast with previous results on negatively charged colloids ${ }^{[13]}$, when the individual ss units hybridize into the ds a large reshaping of their SERS spectra is observed (Fig. 1c), influencing peak position, bandwidths and relative intensity. Notably, if ss1 and ssc spectra show small changes in relative intensity that are associated with the base composition, the stacking and pairing of the nucleobases into the hybridized native structure is revealed by several characteristic features of the Raman melting profiles. $^{[14]}$ Among others, we highlight the marked intensity decrease and peak-shifting of the ring breathing modes (700-800 $\mathrm{cm}^{-1}$ ) and the carbonyl stretching modes $\left(1653 \mathrm{~cm}^{-1}\right)$, the latter one extremely sensitive to the disruption of Watson-Crick hydrogen bonding. ${ }^{[14]}$ We also observe a general narrowing of the Raman features in the $1150-1600 \mathrm{~cm}^{-1}$ spectral region, containing many overlapping bands mainly arising from in-plane vibrations of base residues. ${ }^{[15]}$ Such spectral modification can be ascribed to the adoption of a more rigid and well-defined conformation by the two individual ss when they self-organize into the rigid duplex geometry. The investigation of a set of solutions containing different ds 1 and ssc molar ratios, $R_{\text {hybr }}=[\mathrm{ds} 1] /([\mathrm{ds} 1]+[\mathrm{ssc}])$ (Fig. 1d) shows a progressive shift of the ring breathing bands of adenine (from 734 $\mathrm{cm}^{-1}$ to $730 \mathrm{~cm}^{-1}$ ) when the DNA population is enriched with ds1. The spectral subtraction of the SERS spectra allows us to fully disclose the spectral alterations associated with changes in the DNA structure. In particular, the difference spectrum ds1-ssc (dotted line, Fig. 1d) reveals a minimum at $724 \mathrm{~cm}^{-1}$ and a maximum at $738 \mathrm{~cm}^{-1}$. The corresponding ratiometric peak intensities $I_{724} / I_{738}$ were then selected to monitor the relative ds $1 / \mathrm{ssc}$ populations in the samples, and plotted against $R_{\text {hybr }}$ (Fig. 1e). Data shows that ds sequences can be clearly identified within the mixture even when present at values $<1 \%$, whereas SERS spectra are fully dominated by ds 1 contributions for duplex populations $\geq 20 \%$. Such result is consistent with the higher affinity of dsDNA to AgNP@Sp due to the larger availability of negatively-charged phosphate groups as compared to ssDNA. Linear correlation $\left(r^{2}>0.99\right)$ is observed in the 0.01-0.19 $R_{\text {hybr }}$ range.

To test the possibility of detecting single-base mismatches in DNA duplexes, SERS spectra of the full-complementary ds1 and the heteroduplexes ds2, ds 3 and ds 4 were acquired and compared (Fig. 2). These heteroduplexes contain one adenine base, $A$, in place of: (ds2) one guanine, G, (ds3) one cytosine, C, (terminal position) and (ds4) one cytosine (internal position). Subtraction of the SERS spectra of ds 1 from the other samples generates difference spectra containing vibrational signatures associated with the additional (positive features) and removed (negative features) nucleobase Positive A bands emerge in all difference spectra at 730 and 1507 $\mathrm{cm}^{-1}$, whereas negative $\mathrm{G}$ features are observed at ca. 620,675 and $1354 \mathrm{~cm}^{-1}$ in the ds2-ds1. In this case, negative bands also appear at 1487 and $1577 \mathrm{~cm}^{-1}$, ascribed to purine modes (mainly G contribution) while the other purine feature at $1325 \mathrm{~cm}^{-1}$ (mainly A contribution) does not suffer from a marked change in the relative intensity. These results, together with the lack of significant changes in SERS intensity of the pyrimidine bases, clearly indicate that the spectral alterations are ascribed to the $A \rightarrow G$ base-mismatch in the ds2. On the other hand, ds3-ds1 and ds4-ds1 difference spectra show very similar spectral patterns where, in addition to the positive 


\section{Single-base mismatch}

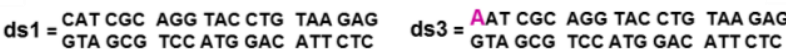
ds2 = CAT CGC AGG TAC CTG TAA GAA $\begin{aligned} & \text { GTA GCG TCC ATG GAC ATT CTC } \\ & \text { ds } 4=\text { CAT CGC AGG TAA CTG TAA GAG } \\ & \text { GTA GCG TCC ATG GAC ATT CTC }\end{aligned}$

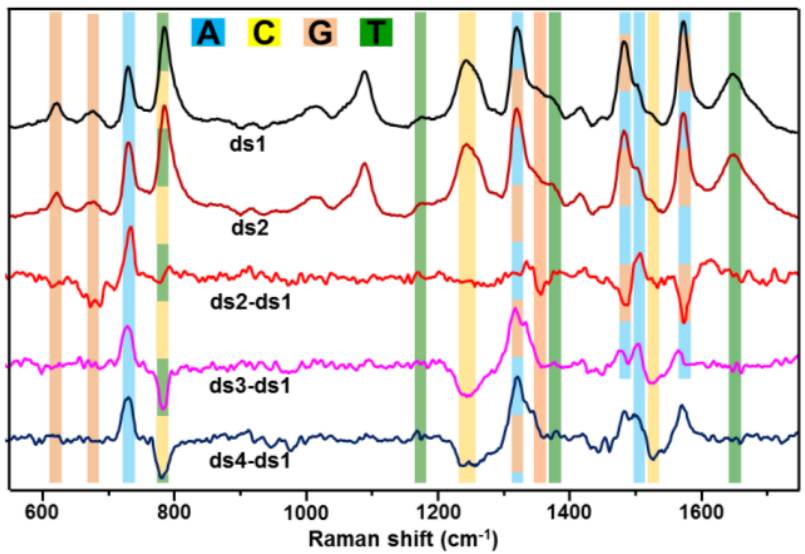

Figure 2. SERS spectra of ds 1 and ds2; and digitally subtracted SERS spectra ds2-ds1, ds3-ds1 and ds4-ds1. For the sake of clarity, the digitally subtracted spectra were multiplied by 3 (for ds2-ds1) and 4 (for ds3-ds 1 and ds4-ds1). Frequency positions of characteristic nucleotide bands are highlighted in: blue (Adenine), yellow (Cytosine), orange (Guanine) and green (Thymine). The overall ds concentration was kept constant at $630 \mathrm{ng} / \mathrm{mL}$.

A $\left(730\right.$ and $\left.1507 \mathrm{~cm}^{-1}\right)$ and the negative $C\left(1250\right.$ and $\left.1528 \mathrm{~cm}^{-1}\right)$ contributions, a consistent intensity increase of the purine bands (1325, 1487 and $1577 \mathrm{~cm}^{-1}$ ) is observed. The pyrimidine ring breathing $\left(787 \mathrm{~cm}^{-1}, \mathrm{C}+\mathrm{T}\right)$ also undergoes a drastic intensity decrease whereas thymine marker bands do not reveal significant alterations. These spectral changes can therefore be associated with the $A \rightarrow C$ base-mismatch in ds3 and ds4. Minor differences between ds3 and ds4 difference spectra (Fig. 2) can be ascribed to the different position of the base mismatch within the sequence. ${ }^{[15]}$

The potential application of AgNP@Sp in SERS analysis of 5methylated cytosine bases $\left({ }^{\mathrm{m}} \mathrm{C}\right)$ and $\mathrm{N} 6$-methylated adenine bases $\left({ }^{m} \mathrm{~A}\right)$ within ds structures was examined by acquiring the SERS spectra of $d s^{m} C$ and $d s^{m} A$ and comparing to their unmethylated analogous ds1. In ds ${ }^{m} C$ and $d s^{m} A$, the $C$ or $A$ nucleobases of one of the strands were all replaced by their respective 5-methylated and N6-methylated counterparts (see sequence structures in Fig. 3 and Fig. 4). All the spectral changes are in good agreement with the normal Raman studies of the substitution of the methyl group into the $5 \mathrm{C}$ and $\mathrm{N} 6$ atom in cytosine ${ }^{[16]}$ and adenosine ${ }^{[17]}$ nucleosides. The major spectral markers of the cytosine methylation can be easily recognized through the whole difference spectrum (Fig. 3a). Among others, we observe a red-shift and relative intensity decrease of the pyrimidine ring breathing band at $787 \mathrm{~cm}^{-1}$, together with the appearance of a new weak feature at ca. $755 \mathrm{~cm}^{-1}$. In the 1200-1450 $\mathrm{cm}^{-1}$ spectral region, the normal Raman of unmodified cytidine aqueous solution contains two main bands at 1242 and $1292 \mathrm{~cm}^{-1}$, whereas the 5-methyl substituted shows a very different spectral signature with three discernible bands at 1224, 1258 and $1302 \mathrm{~cm}^{-1}$ and a new very intense feature at $1362 \mathrm{~cm}^{-1} \cdot{ }^{[16]}$ Accordingly, the difference spectrum in Fig. 3a reveals two negative bands at ca. 1244 and $1287 \mathrm{~cm}^{-1}$; three positive contributions broadly centered at 1218, 1268 and $1315 \mathrm{~cm}^{-1}$, and a very strong and sharp band at $1362 \mathrm{~cm}^{-1}$. Moreover, the $\mathrm{C}$ ring band at ca. $1510 \mathrm{~cm}^{-1}$ also undergoes a marked red-shift and intensity decrease while the broad carbonyl stretching band centered at $1653 \mathrm{~cm}^{-1}$ shows a large red-shift (well highlighted in the difference spectrum by the negative feature at ca. $1622 \mathrm{~cm}^{-1}$ and the positive one at ca. $1662 \mathrm{~cm}^{-1}$ ).
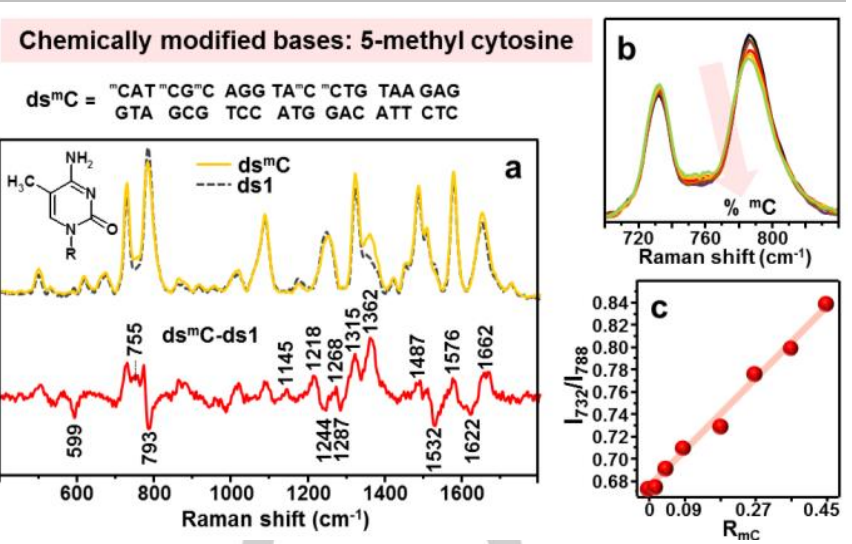

Figure 3. (a) Molecular structure of 5-methyl Cytosine $\left({ }^{\mathrm{m}} \mathrm{C}\right)$. SERS spectra of $\mathrm{ds} 1$ and $d s^{\mathrm{m}} \mathrm{C}$, and the difference spectrum $\mathrm{ds} \mathrm{m}^{\mathrm{m}} \mathrm{C}-\mathrm{ds} 1$. For the sake of clarity, the digitally subtracted spectra were multiplied by a factor of 2. (b) Detail of the $700-840 \mathrm{~cm}^{-1}$ region for the SERS spectra of $\mathrm{ds} 1+\mathrm{ds}^{\mathrm{m}} \mathrm{C}$ at different cytosine base ratios $R_{m c}=\left[{ }^{m} C\right] /\left([C]+\left[{ }^{m} C\right]\right.$ ) (from the top to the bottom, $R_{m c}=0 ; 0.045$; $0.091 ; 0.182 ; 0.273 ; 0.364$; and 0.455$)$. The overall ds concentration was kept constant at $630 \mathrm{ng} / \mathrm{mL}$. (c) Ratiometric peak intensities $\mathrm{I}_{732} / \mathrm{l}_{788}$, vs. the molar ratio $R_{m c}=\left[{ }^{m} C\right] /\left([C]+\left[{ }^{m} C\right]\right)$.

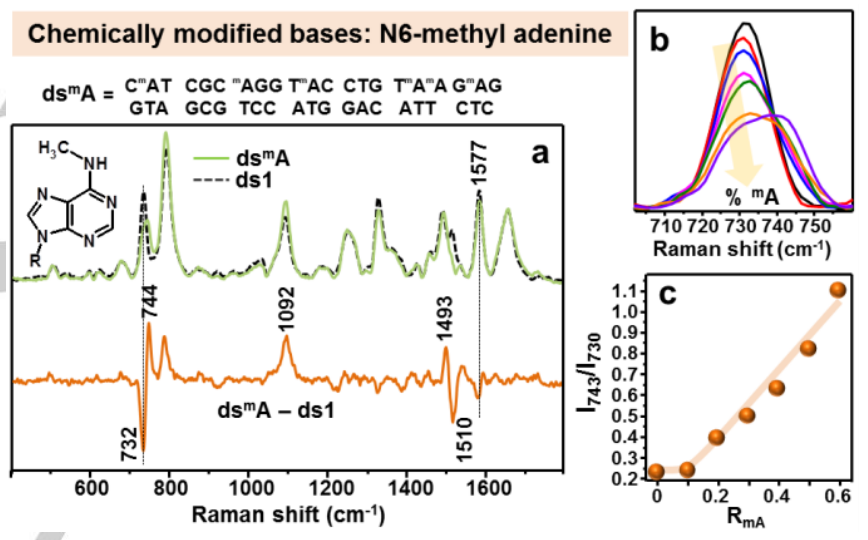

Figure 4. (a) Molecular structure of N6-methyl Adenine $\left({ }^{m} A\right)$. SERS spectra of $d s 1$ and $d s^{m} A$, and the difference spectrum $d s^{m} A-d s 1$. For the sake of clarity, the digitally subtracted spectra were multiplied by 2. (b) Detail of the 700-760 $\mathrm{cm}^{-1}$ region for the SERS spectra of $d s 1+d s^{m} A$ at different adenine base ratios $R_{m A}=\left[{ }^{m} A\right] /\left([A]+\left[{ }^{m} A\right]\right.$ ) (from the top to the bottom $R_{m A}=0 ; 0.1 ; 0.2 ; 0.3 ; 0.4 ; 0.5$ and 0.6). The overall ds concentration was kept constant at $630 \mathrm{ng} / \mathrm{mL}$. (c) Ratiometric peak intensities $I_{743} / l_{730}$, vs. molar ratio $R_{m A}$.

The spectral modifications of the SERS spectral profile of the normal DNA upon methylation of six of their eleven the $A$ bases are also striking (Fig. 4a, difference spectrum). The $A$ ring breathing band at $731 \mathrm{~cm}^{-1}$ undergoes a drastic $11 \mathrm{~nm}$ up-shift together with a notable intensity decrease, whereas the pyrimidine stretching of the adenine at $1509 \mathrm{~cm}^{-1}$ almost disappears from the spectrum. Furthermore, we observe up-shifts of the phosphate stretching mode at $1090 \mathrm{~cm}^{-1}$ (together with a marked intensity increase); the $\mathrm{v}(\mathrm{CN})$ imidazole feature at $1487 \mathrm{~cm}^{-1}$ and the $1577 \mathrm{~cm}^{-1}$ band ascribed to base ring modes (mainly $\mathrm{G}+\mathrm{A}$ ).

Quantification of relative methylated base populations within the sample was investigated by monitoring the ratiometric peak intensities $\mathrm{I}_{732} / \mathrm{l}_{788}$ for ${ }^{\mathrm{m}} \mathrm{C}$ and $\mathrm{I}_{743} / \mathrm{l}_{730}$ for ${ }^{\mathrm{m}} \mathrm{A}$, at different molar ratios $\left(R_{m c}=\left[{ }^{m} C\right] /\left([C]+\left[{ }^{m} C\right]\right)\right.$ and $\left.R_{m A}=\left[{ }^{m} A\right] /\left([A]+\left[{ }^{m} A\right]\right)\right)$. Details of the ring breathing spectral regions are illustrated in Fig. $\mathbf{3 b}$ and $\mathbf{4 b}$. In the case of $d s^{m} \mathrm{C}$ (Fig. $\mathbf{3 b}$ ), we highlight the progressive intensity decrease of the $\mathrm{C}+\mathrm{T}$ ring breathing band at $787 \mathrm{~cm}^{-1}$ upon increasing of the relative ${ }^{\mathrm{m}} \mathrm{C}$ populations. Differently, for $\mathrm{ds}^{\mathrm{m}} \mathrm{A}$ (Fig. 4b), the $A$ ring breathing band at $730 \mathrm{~cm}^{-1}$ suffers a dramatic 
intensity decrease and large peak red-shift as the $R_{m A}$ value becomes larger. Linear correlations were obtained in both cases $\left(r^{2}\right.$ $>0.99$ for ${ }^{\mathrm{m}} \mathrm{A}$ and $>0.94$ for ${ }^{\mathrm{m}} \mathrm{C}$ ) with detection limits of one ${ }^{\mathrm{m}} \mathrm{C}$ per 22 total cytosine bases and one ${ }^{\mathrm{m}} \mathrm{A}$ per 10 total adenine bases in the sample (Fig. 3c and 4c, respectively).

In summary, we have demonstrated the potential application of SERS for the development of a fast and affordable high-throughput screening method for gaining detailed genomic information on DNA duplexes. Importantly, due to the low amount of DNA required (comparable to only 10-100 cells), the analysis can be performed without the necessity of amplification thus providing realistic direct information of the DNA in its native state. Due to the direct nature of this sensitive and selective assay, we anticipate these findings of key importance in a number of different fields including diagnosis, genetic engineering, biotechnology, drug discovery (antibiogram in microbiology), agriculture and forensic science. ${ }^{[1,18]}$

[1] a) L. Pikor, K. Thu, E. Vucic, W. Lam, Cancer Metastasis Rev. 2013, 32 , 341-352; b) S. P. Jackson, J. Bartek, Nature 2009, 461, 1071-1078.

[2] a) S. Yang, R. E. Rothman, Lancet Infect. Dis. 2004, 4, 337-348; b) A. Git, H. Dvinge, M. Salmon-Divon, M. Osborne, C. Kutter, J. Hadfield, P. Bertone, C. Caldas, RNA-Publ. RNA Soc. 2010, 16, 991-1006.

[3] X. Qiu, L. Wu, H. Huang, P. E. McDonel, A. V. Palumbo, J. M. Tiedje, J. Zhou, Appl. Environ. Microbiol. 2001, 67, 880-887.

[4] E. E. Schadt, S. Turner, A. Kasarskis, Human Molecular Genetics 2010 19, R227-R240.

[5] a) J. N. Anker, W. P. Hall, O. Lyandres, N. C. Shah, J. Zhao, R. P. Van Duyne, Nat. Mater. 2008, 7, 442-453; b) K. Saha, S. S. Agasti, C. Kim, X. N. Li, V. M. Rotello, Chem. Rev. 2012, 112, 2739-2779.

[6] a) R. A. Alvarez-Puebla, L. M. Liz-Marzan, Small 2010, 6, 604-610; b) W. Xie, S. Schlücker, Phys. Chem. Chem. Phys. 2013, 15, 5329-5344.

[7] D. Graham, K. Faulds, Chem. Soc. Rev. 2008, 37, 1042-1051.

[8] A. Barhoumi, D. Zhang, F. Tam, N. J. Halas, J. Am. Chem. Soc. 2008, 130, 5523-5529.

[9] a) A. Barhoumi, N. J. Halas, J. Phys. Chem. Lett. 2011, 2, 3118-3123; b) S. R. Panikkanvalappil, M. A. Mackey, M. A. El-Sayed, J. Am. Chem. Soc. 2013, 135, 4815-4821; c) S. R. Panikkanvalappil, M. A. Mahmoud, M. A. Mackey, M. A. El-Sayed, ACS Nano 2013, 7, 7524-7533; d) E. Papadopoulou, S. E. J. Bell, Angewandte Chemie (International ed. in English) 2011, 50, 9058-9061; e) Ž. Krpetić, I. Singh, W. Su, L. Guerrini, K. Faulds, G. A. Burley, D. Graham, J. Am. Chem. Soc. 2012, 134, 8356-8359.

[10] D. van Lierop, Z. Krpetic, L. Guerrini, I. A. Larmour, J. A. Dougan, K. Faulds, D. Graham, Chem. Comm. 2012, 48, 8192-8194.

[11] F. J. García de Abajo, Reviews of Modern Physics 2007, 79, 12671290.

[12] R. Aroca, Surface-enhanced Vibrational Spectroscopy, John Wiley \& Sons, Chichester, 2006.

[13] a) E. Papadopoulou, S. E. J. Bell, Chem.-Eur. J. 2012, 18, 5394-5400; b) N. E. Marotta, K. R. Beavers, L. A. Bottomley, Anal. Chem. 2013, 85, 1440-1446.

[14] J. G. Duguid, V. A. Bloomfield, J. M. Benevides, G. J. Thomas, Biophys. J. 1996, 71, 3350-3360.

[15] H. Deng, V. A. Bloomfield, J. M. Benevides, G. J. Thomas, Biopolymers 1999, 50, 656-666.

[16] A. Gfrorer, M. E. Schnetter, J. Wolfrum, K. O. Greulich, Ber. BunsenGes. Phys. Chem. Chem. Phys. 1991, 95, 824-833.

[17] S. Mansy, W. L. Peticolas, R. S. Tobias, Spectroc. Acta Pt. A-Molec. Biomolec. Spectr. 1979, 35, 315-329.

[18] a) G. H. Clever, M. Shionoya, Coord. Chem. Rev. 2010, 254, 2391 2402; b) C. J. Lord, A. Ashworth, Nature 2012, 481, 287-294.

\section{Acknowledgements}

This work was funded by the Spanish Ministerio de Economia y Competitividad (CTQ2011-23167), the European Research Council (CrossSERS, FP7/2013 329131, PrioSERS FP7/2014 623527) and
Medcom Advance SA. We are grateful to Dr. Matteo Masetti (Department of Pharmacy and Biotechnology, Universitá di Bologna, Bologna, Italy) for the dsDNA drawings.

Keywords: DNA $\cdot$ SERS $\cdot$ hybridization $\cdot$ single-base mismatch - methylated bases

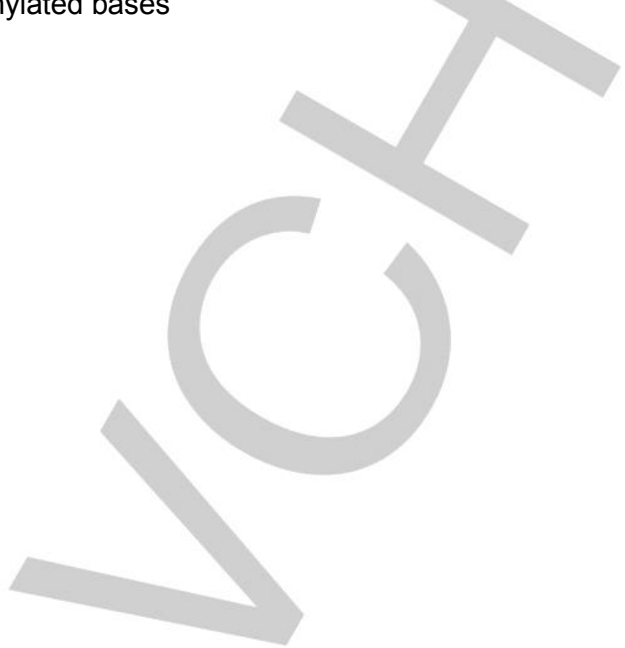




\section{Entry for the Table of Contents}

\section{COMMUNICATION}

Accessing genomic information.

Fast and affordable high-throughput screening method based on SERS as a low-cost and ultrasensitive genotyping strategy for gaining detailed genomic information on DNA duplexes: recognition of hybridization events, single-base mismatch and base methylation.

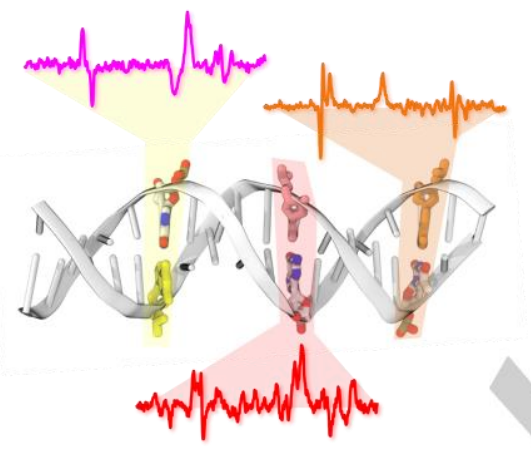

Luca Guerrini, “ Željka Krpetić, Danny van Lierop, Ramon A. Alvarez-Puebla and Duncan Graham.

Page No. - Page No.

Direct Surface-enhanced Raman Scattering analysis of DNA duplexes 